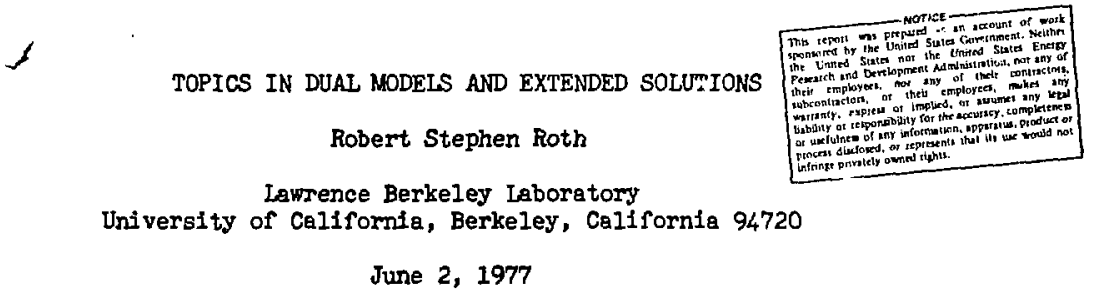

\begin{abstract}
Two main topics are explored. The first deals with the infintiles arising from the one loop planar string diagram of the ste:dard dual model. It is shown that for the number of dimensions $d=25$ or 26, these infinfties lead to a renormalization of the slopt of the Regge trajectories, in addition to a renormalization of the coupling constant. The second topic deals with the propagator for a confined perticle (monopole) in a rleld theory. When summed to all orders, this propagator is altogether free of singularities in the finfte momentur plane, and an attempt is made to 1liustrate this. We examine the Bethe-Salpeter equation and show that ladder diagrams are not sufficlent to obtain this result. However, in a nonrelativistic approximation confinement is obtained and all poles disappear.
\end{abstract}

* This work supported by the U. S. Fnergy Research and Development Administration under the ausplces of the Depertment of Physical Research. 
TABLE OF CONTENTS

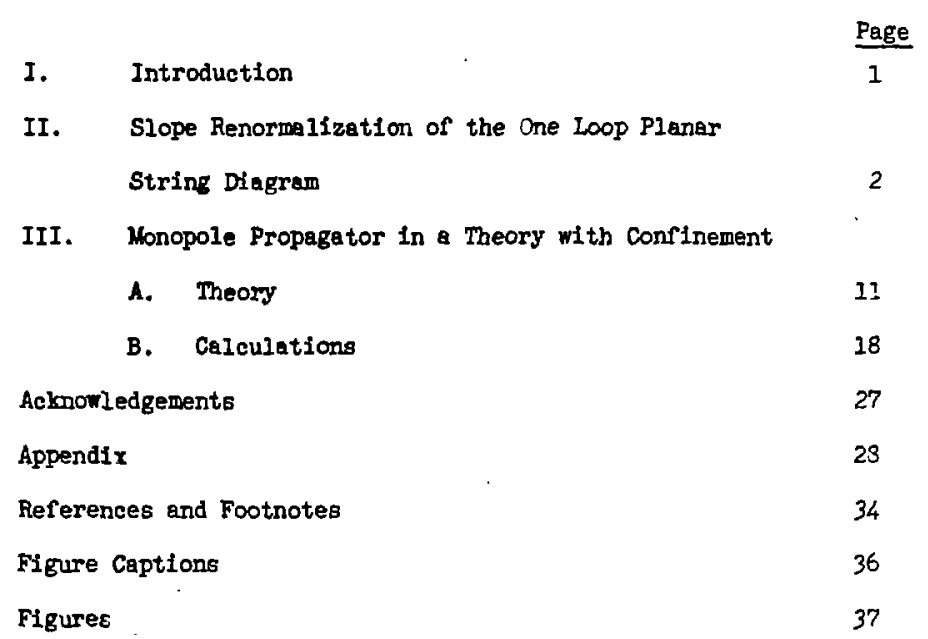


I. INTRODUCTION

The two major approaches to elementary particle physics in recent years have been quantum field theory and S-matrix theory. The former tries to derive physically relevant quantities from interactions of relativistic quantum fields. The lat.ter insists that only the scattering amplitude is physicaliy important. It then tries to derive what it can from properties it demands of the S-matrix. Dual models started out in the radical fing of the S-matrix camp. The original Veneziano formula ${ }^{1}$ Fas proposed as simply an example of a scattering amplitude that satisfied certain criteria. At present dual models stend almost on the borderline between s-matrix and field theories, with many morkers trying to obtain the extended structures encountered in dual models from bona fide field theories. In this thesis we present two pieces of work. In Part I, a proof is given that in the simplest dual model, divergences can be renormalized at the one loop level. In Part II, a detalied examination of the monopole propagator is given for a theory with confined monopoleentimonopole pairs. This theory is relevant to dual models in the sense that the otring of magnetic flux connecting the monopole-antimonopole pair is a linear extended structure similer to the dual string. 
II. SLOPE RENORMALIZATION OF THE ONE TWOPP

PLANAR STRING DIAGRAM

Numerous excellent review articles in dual models have appeareo in the literature. ${ }^{2}$ He present here only a brief sumary of some of the major developments in the field as an introduction. Veneziano's four point function ${ }^{l}$ was soon generalized to $n$ particles by a number of authors. 3 By factorization, this gave the complete S-matrix. This S-matirx has Inear Regge trajectories, Regge esymptotic behavior, auaitity, factorizetion, and the statistical model density of statis. The particles have zero width, but it was hoped that higher order corrections would cure this problem. After this an operatpri formelism was developed ${ }^{4}$ in which factorization is apparent, and ghosts were proved to decouple. 5 Next it was realized that the whole formalism is equivalent to the quantum mechanics of Inassless relativistic strings. 5,6 Interactions are introduced as the splitting and joining of strings and the amplitude can be determined totalily by the topology of the string diagram.? In particular, the amplitude equals the functional average of exp (1 $\times$ Action) for all $X^{\mu}(\sigma, \tau)$ satisfying the boundery conditions of the string diegrem. Higher order corrections can then naturally be obtained from string diagrams with loops. The planar loop gives the resonences widths, as had been hoped, but the nonplanar loop generates the Pomeron trajectory, which has twice the Intercept and half the slope of the ordinary Regge trajectories. Before going on to a discussion of 
renormalization, we mention the two mafor defects of the model that we have so far been sweeping under the rug. First, the theory is only consistent in an unhysical number of dimensions, 26 for the original model, 10 for another (Neven-Schwarz" rodel ${ }^{9}$ wth a spinning string. Secondly, the intercepts are too high, I for the ordinary Regge trajectories, 2 for the Pomeron trajectory,

In duel nodels, infrared divergences appear due to the preserce of zero mass particles. For the number of dizmsions less than 25, Neven and Scherk ${ }^{10}$ proved that the infinities of the planar loop can be absorbed into a renorzalization of the coupling constant. The case of dimensions 25 or 26 is treated in the following paper, where it is shown thet the infinities lead to a renomalization not only of the coupling constant, but also of the slope of the Regge trajectories. 


\title{
Slope renormalization of the one-loop planar string diagram*
}

\author{
Robert Roth \\ Lawrence Berkeley Loborotony, University of California, Berkeley, Colijomia 94720
}

(Received 24 November 1975)

\begin{abstract}
It has been previously thown that for the number of dimensions $d<25$, the one-loop planar string diagram is simply a multiplicative (coupling constant) renormslizetion of the Born term. $\mathbf{H}$ is shown bete thes for $d=25$ or 26 the exirn divergent term give, in sodition 10 a further multiplicative renormatiztion, a remonmalization of the slope of the Regle trajectories.
\end{abstract}

\section{INTRODUCTION}

The $\boldsymbol{N}$-point Veneziano amplitude is a treediagram approximation to the strong-interaction ecattering amplitute. Thus, one is ted to examine loop diagrams in the hope of obtaining a more realistic amplitude. As in quantum electrodymamics. theae diagrams are found to be divergent. A renormalization procedure must then be found in which all infinities are absorbed into a redetInition of the physical parameters of the theory. This was done for the one-loop planar diagram for d<25 by Neveu and Scherk, ${ }^{1}$ and the result was found to be a simple multiplicative renormalization of the Boru term. In this paper we do this for the one-loop planar diagram in the critical number of dimensions. We ahow that the divergent part of the amplitude can at the one-loop level be written as

$$
c_{1}\left(B_{N}\left(m, s_{i j}\right)+c_{2} \frac{d B\left(m, s_{1}\right)}{\partial m}\right)=c_{1} B_{N}\left(m+c_{2}, s_{d}\right)
$$

where $c_{1}, c_{2}$ ale constants, $s_{1}$ are the planar Bubenergies, $m$ is the slope of the Regge traJectorisa, ant $B_{N}$ is the Veneziano amplitude. Thus, at this level, multiplicative and slope renormalizations are all that are needed to render the integral fintte.

\section{METHOD}

In the Interacting-string picture, the singleloop planar amplitude for $\mathbf{N}$ scalars is given by the following expression, after a Jacobi transformation has been made on the usual variables of integmtionit:

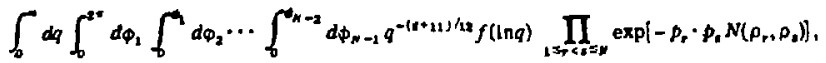

where

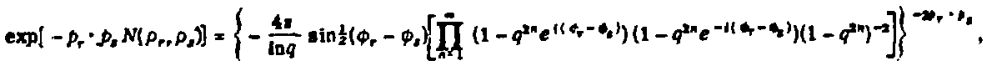

and where $f(\ln q)$ is a function of ing (no powers), $\phi_{n}=0$, and the factor $q^{-(\alpha+11) / h t}$ should read intead $q^{-3}$ for the special case $d=26$. The intearal diverges near $q=0$, that is, the region where the loop shrinks to a point. We note for later use that this point (the loop at $q=0$ ) is located at $i \infty$ in the $\varphi$ plane. To examine the integral in the $q=0$ reglon, ve expand the expression in large equare brackets in a power enerles in $q^{2}$ and find that for $d<25$, only the constant term leads to a divergent integral. This term was shown by Neveu and Scherk ${ }^{1}$ to be simply a multiplicative renormalization of the Veneztano amplitude. For $d 225$, the linear term in $q$ in the power-series expansion also leads to a divergent integral. It is this integral that we examine here. We change to the more convenient variables of integration $u_{1}=\tan \xi \varphi_{1}$. In the limit $q-0$, these $u$ variables are related to the string-diagram variables $\rho$ by the usual tree-diagram transformation

$$
\rho=\sum_{=1}^{4} a_{r} \ln \left(u-u_{r}\right)
$$

$w$ ith the cut now at $u=i$. We then obtain as the coesidicient of the infinite $q$ integration

Reproduced with permission from Physical Review D, Brookhaven flational Laboratory Upton, New York 11973 


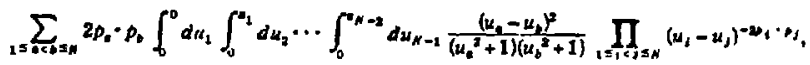

where the range of integration is restricted only by $\omega_{N}=0<u_{N-1}<\cdots<u_{1}<0=w_{N}$ (which is just a convenient notation for $0<\mu_{N-2}<\cdots<\mu_{r}<0$ and $-\infty<\mu_{r-1}<\cdots<u_{1}<0$ for some $\left.r\right)$.

Even after factoring out the infinite o integral, we find that the remaining integral (3) diverges. This remaining divergence cannot sull be due to the loop shrinking to a point since the integral (3) diverges only for particular configurations of the $u^{\prime} \mathrm{s}$. In fact, the remaining divergence is due to configurations of the one-loop diagram that correspond to external-line self-energy insertions. Suppose we factor out the elf-energy part in one of these configurations. Then we are left with a tree-level diagram with exactly the ame incoming states and momenta, or else the contribution is not divergent. Thus, we expect our divergences to be simply an infinite constant multiple of $B_{y}$.

In order to evaluate the contribution of $(3)$ to the amplitude, we must first choose come cutorf procedure rendering the integral tinite. To this end, we temporarily suspend momentum conservation by introducing a new incoming momentur $h$ (see Fig. 1). We tentatively choose it to ente. the string diagram at the position of the loop (remember that we have taken the Imil $q-0$ wtich corresponds to the loop shrinking to a point), but we shall see that we will have to modify this slightly. We expect this procedure to eliminate out infinfties, oince now all self-energy insertions have incoming and outgoing momenta which differ by $k$. In the limit $k-0$, we should then recover a constant multiple of $B_{N}$ as the divergent part. Thus, instead of the normal energy-momentum conservation equation, we have

$$
\sum_{1=1}^{k} p_{1}+k=0
$$

where $k$ is the new momentum introduced.

This new momentum introduces an extra term

$$
\left.i \sum_{i}^{1} x^{\prime} \omega_{c}\right)
$$

to the exponential of the functional integral for the $S$ matrix. This leads to the extra term in (1)

$$
\exp \left[\sum_{i=1}^{\infty} \sum_{i=1}^{h} k_{1} p_{c}^{\prime} N\left(p_{r}, p_{c}\right)+\frac{1}{2} N\left(p_{c}, p_{c}\right) \sum_{i=1}^{2} k_{i}^{2}\right] .
$$

The second term, which in infinite, is similar to an infinite term obtained in the conventional path-integral interacting-string formalizm. As in the latter case, it can be absorbed into the volume element since it has no dependence on the integration variables. The first term in the exponent changes (3) to the expression

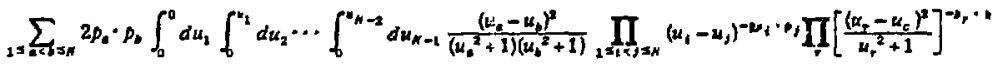

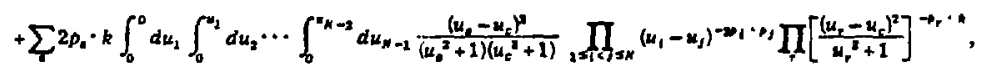

where we have defined $k_{1}=0$ to change the $d-2$ product to a covariant d product, $u_{t}=i$ is the point to which the loop has been mapped, and we have neglected terms in $k^{*}$ since they are eccond order in a 5 mall quantity. The fact that $\left(u_{c}{ }^{2}+1\right)^{-1}$ $=\left(\hat{t}^{2}+1\right)^{-1}$ appearing in the second term in undefined is a point we shall deal with later. We can write the last factor of the first term in (4) as

$$
\begin{aligned}
\prod_{p}\left[\frac{\left(u_{r}-u_{c}{ }^{2}\right.}{u_{r}^{2}+1}\right]^{*_{r} \cdot 1} & =\exp \left[-\sum p_{r} \cdot k \ln \left(\frac{\left(u_{r}-u_{c}\right)^{2}}{u_{r}^{2}+1}\right)\right] \\
& =1-\sum p_{r} \cdot k \ln \left(\frac{\left(u_{r}-u_{c}\right)^{2}}{u_{r}^{2}+1}\right) \\
& +O(k)
\end{aligned}
$$

This expansion ts valid in the range of integration since the $u_{p}{ }^{\prime} s$ are real and $u_{\varepsilon}=i$, go that the argu- 


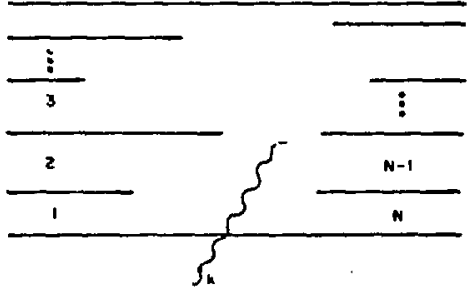

FJG. 1. N-point function with oww momentum h ederm.

ment of the logarithm never blows up. Alsc aince the logarithm is always well behaved the convergence properties of all the terms in the series are the same. Thus, since we will see that the first term in the series behaves as $k^{-1}$ as $k \rightarrow 0$ (this behavior is expected of an external-line sellenergy insertion), we can neglect the terms of order $k^{2}$ and higher in (5). Thus, we have left the first two terms in the expansion (5) in addition to the second term in (4). We refer to these throughout the rest of this paper as terms 1, II, and III. We point out that although term I has exactly the same form as the original divergent expression (3), It is now well behaved due to the ew energy-momentum conservation equation.

\section{I. EVALUATION OF THE N.POINT FUNCTION}

Consider term I for the $N$-point function for a particular cholce of $a, b * N$ and $w_{a}<0$ (just as an (Ilusiration). Then we have

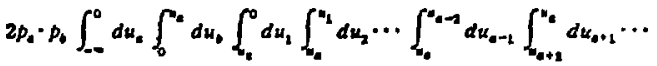

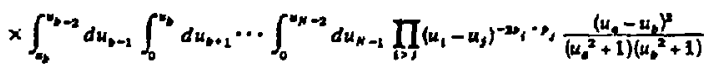

$$
\begin{aligned}
& =2 p_{0} \cdot p_{0} \int_{-=}^{0} \frac{d u_{c}}{u_{0}^{2}+1} \int_{0}^{-1} \frac{d \alpha_{2}}{\left(\alpha_{0}^{2} u_{0}^{2}+1\right)}\left(1-\alpha_{3}\right)^{2} \int_{-1}^{0} d \alpha_{1} \int_{-1}^{\alpha_{1}} d \alpha_{2} \cdots
\end{aligned}
$$

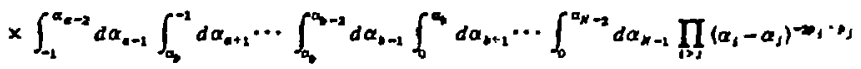

$$
\begin{aligned}
& =x p_{0} \cdot p_{0}\left(\int_{0}^{1}+\int_{n}^{n-1}+\int_{n-1}^{\infty}+\int_{-n}^{-1}+\int_{n-1}^{-1}\right) d a_{0} \frac{\left(1+a_{0}\right)^{2}}{1+\left|\alpha_{n}\right|}
\end{aligned}
$$

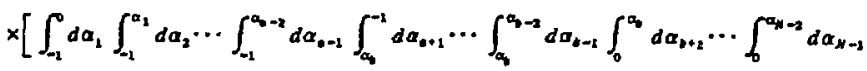

$$
\begin{aligned}
& \left.\times \prod_{i=1}\left(a_{1}-\alpha_{j}\right)-\omega_{i} \cdot n_{j}\right] \text {. }
\end{aligned}
$$

In (6a), we have made the substitution $\alpha_{1}=z_{1} / u_{0}$. In (6b), we have done the $u_{a}$ integration and broken up the $\alpha_{3}$ integral as shown. If all the $\epsilon_{1}$ were zero, then the quantity in the square brackets would be $\left[1 / a_{d}\left(1+a_{b}\right)\right] B_{N}$, where $B_{k}$ is the $N$-point Venezino (Koba-Nielsen) formula. In the limit $\epsilon_{1}-0$, the secone and fifth $\alpha_{b}$ integrals are still finite. Thus, we are permitted to take the limit before doing the $\alpha_{b}$

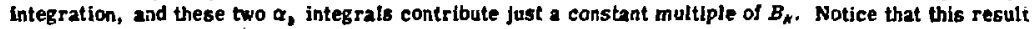
is independent of the value of $\eta>0$, and we can choose it to be as small as we like. In particular, we can let $\eta \rightarrow 0$, as long as this limit is taken ofter the $c_{1}-D$, and we choose to do so for convenience.

Now let us examine the first $\alpha$, integration. Since the range $\alpha_{\text {. }}$ ne $\alpha_{b}$ integration is infinitesimal, the only possible contribution to this term can arise when the integrand blows up for $\epsilon_{d}=0$. This occurs only when all but one of the $u$ 's are equal. A detailed calculation for several $N$ confirms this, but we know this must be the case in general since this region corresponds to the configuration where the loop is in one of the stringe and far from the interaction region. Since we atready havi $a_{b}=u_{b} / u_{t}=0$, the only possibility for the $u$ 's in which all but one are equal is $u_{1}=0$, $i \neq a$. Thus, we can restrict the other $\alpha$ 's to be less than some number $\xi$, where we can clearly choose $\pi \ll \xi \ll 1$. In fact, after a littie thought, it is 
clear that, in addition to the above inequality, we can lake $\xi$ as small as we like, by simultaneously making $\eta$ smaller if necessary. Dolng this, we obtain

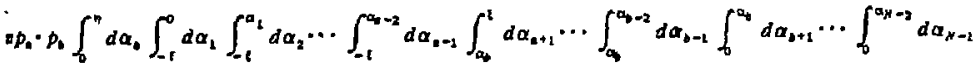

$$
\begin{aligned}
& \times \prod_{i, j=0}\left\langle a_{i}-\alpha_{j}\right\}^{-\nu_{i} \cdot n_{1}}
\end{aligned}
$$

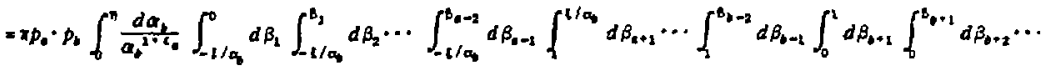

$$
\begin{aligned}
& \times \int_{0}^{B_{N-2}} d \beta_{N-1} \prod_{i, j=1}\left(\beta_{1}-\beta_{1}\right)^{-2 \nu_{1} \cdot \beta_{1}}
\end{aligned}
$$

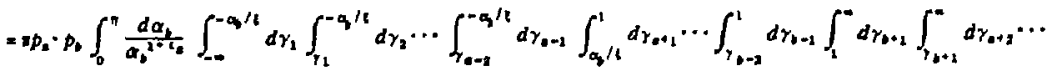

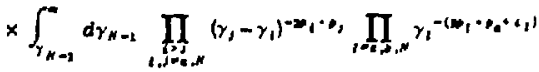

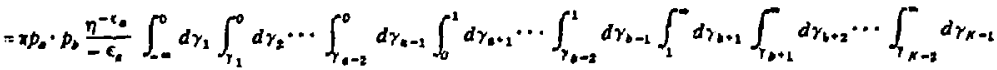

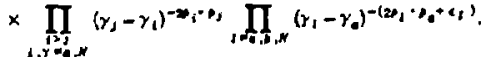

In $(7 a)$ we have excluded those factors wilh $i$ or $j$ equal to $a$ since $\alpha_{a}=1$ and all other $\alpha_{i}=0$. Equa tion (7b) is obtained by the substitution $\beta_{i}=\alpha_{1} / \alpha_{0}$ and $(7 c)$ by $\gamma_{1}=\beta_{1}^{-1}$, and where we have used the altered energy-momentum condition extensively. Equation (7d) follows only if the extra pieces added (by changing the limits of integration) contribute nothing to the integral. This will oceur only if $s_{, 0}<-2, j=1, \ldots, a-1$ and $s_{k}<-2$, $1=a+1, \ldots, b-1$, where $s_{a b}=\left(b_{a}+p_{a+1}+\cdots+p_{b}\right)^{2}$.

It is quite tempting to identify (7b) immediately as a linear combination of derivalives of $B_{\mu}$ vith respect to $p_{i} \cdot p_{l}, l \neq a_{,} b, N$. However, this is quite migleading, wince the $p_{1} \cdot p$, are not all independent due to the $N$ relations

$$
p_{i} \cdot\left(\sum_{i=1}^{\pi} p_{i}+k\right)=0 \text {. }
$$

If we were to use these relations to eliminate $N$ of the $\mu_{1} \cdot p_{J_{1}}$, then the products $p_{a} \cdot p_{1}, l * a, b, N$ would appear elsewhere, and our simple argument would break down.

In order to see that ( $7 d$ does involve the deriva- tive of $B_{N}$, It will be convenient to change to the variables $s_{a b}$ defined above. These have the ad. vantage that they are all independent (we count. $s_{l,-1}=s_{, N}$ as one, etc.), unlike the $p_{1} \cdot p_{j}$ which are restricted by (8). We must however decide where we will put $k$ in the defintion of the $s$ al. That is, we could choose

$$
\begin{aligned}
s_{a l} & =\left(p_{a}+p_{a+1}+\cdots+p_{b}+k\right)^{2} \\
& =\left(p_{L_{1}}+p_{a_{2}}+\cdots+p_{a-1_{1}}\right)^{2}
\end{aligned}
$$

or we could put the $k$ in the last expression. We note that using the wrong $s_{a}$ in the divergent term leads to extra finite terms in the final result. Since the divergent part of the term we are dealing with here is proportional to $\left(1 / \epsilon_{*}\right) B_{H}$, it arises from the con'iguration where the loop is in string $a$. Thus, the arguments of the $B_{\mu}$ should be the kinematic variables with $p_{a}$ replaced by $p_{a}+k$. We therefore use the $s^{*}$, delined so that the $k$ appears in that sum of momenta that contains $p_{a}$. Then it is not hard to show (working backwards) that

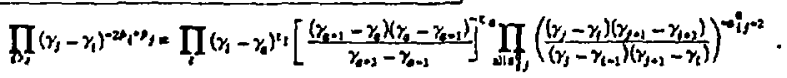


If one of the $\gamma^{\prime} s$ is infinits (here $\gamma_{\gamma}$ ), then this formula stj]l holds and all factors with that $\gamma$ cancel. Also we can write the aquare bracket in (8) as

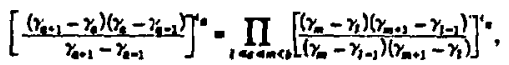

where the above result depends crucislly on the relations $\gamma_{a}=0, \gamma_{b}-1, \gamma_{c}=\infty$. Then using $(\theta)$ and $(10)$, (7d) becomes

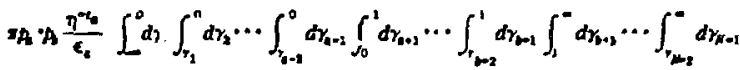

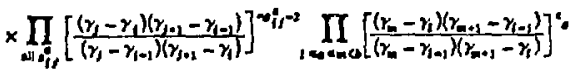

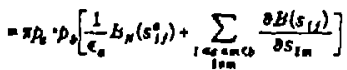

In the limit $\epsilon_{\varepsilon}-0$. Terms proportional to Inך have been dropped, since it is known that they cancel with terms in otlinr 0 , jntegrais (this is due to the lact that $\eta$ is an arbitrary division polnt of an integral). Notice that it is unimportant which $s_{U}$ 's we use as the ergument of the derivatives of $B_{K}$ in the limit $\epsilon_{\mathrm{f}}-0$.

By a cimilar argumeni, the third and fourth $\alpha_{b}$ integrals each lead to an identical expregsion to (Jd) except that $c_{p}$ replaces $\epsilon_{d}$ in the coefficient, and analogous steps lead to an expression similar to (11). If $u_{*}>0$ we get, in addition to (11), one more term identical to (11). Although the calculation is stightly dj' ferent. the result holds over if one of $a$ or $b$ equals $N$. However, in order to obtain (7d) for all $a, b$, we must have $s_{a s}<-2$ for all planar channels. This poses no problem as the $s_{a b}$ are all independent. Later the proof holds also in the physical region by analytic continuation.

Adding up all the contributions, we obtain for term 1

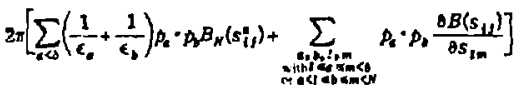

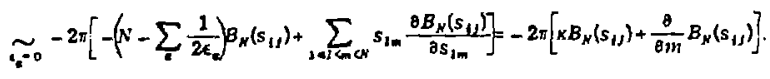

where $*$ is a constant, $m$ is the slope, and we have used the relations

$$
2 p_{a} \cdot p_{b}=s_{a b}-s_{a, b+1}-s_{a+1, b}+s_{a+1, b-1}
$$

(in which we must use the definitions $s_{\omega}=-1$, $s_{\text {mot }}=0$ ). This is the desired result, and thus we have a universal renormalization of the slope of the Regge trajectories..

To compiete thie proof, we must show that terms $I I$ and III do not affect our resuit. Term II is acwally a sum of terms with the term I integrand and the estra pieces

$$
-\sum \frac{c_{c}}{2} \ln \left[\frac{\left(u_{e}-i\right)^{2}}{u_{e}^{2}+1}\right] .
$$

Since the extra term is well behaved throughout the range of integration and is first order in $\epsilon_{f t}$ we neglect alt but the divergent part of the integral. If $* \neq a$ or $b$, then this always occurs for $u_{r}=0$, and we can write

$$
\frac{\epsilon_{r}}{2} \ln \left[\frac{\left(u_{r}-i\right)^{2}}{u_{r}^{2}+1}\right]=-\frac{i \pi \epsilon_{r}}{2},
$$

and the result is a multiple of the divergent part for $d<25$. For $r=a$ or $b$, we write

$$
\ln \frac{\left(u_{r}-t\right)^{2}}{u_{r}^{2}+1}=-2 i \tan ^{-t} \frac{1}{u_{r}}
$$

Then the $u_{n}$ integration is modifiud using

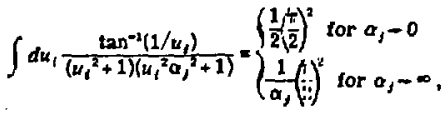

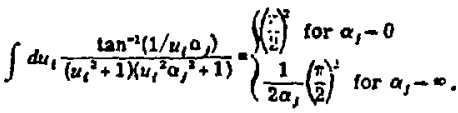

Adding all the terms up, we find that term It is proportional to 


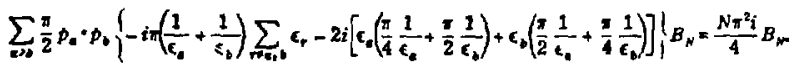

As we remarked earlier, term $I I$ contains the explicit factor $\left(i^{2}+1\right)^{4 t}$ which must be removed. This can be done by displacing the point of entry of the new momentum to a lixed point in the string diagram infinitesimally close to the loop. Since we are dealing with the case where the loop has shrunk ta a point, we can use the tree-dia- gram transformation (2) to find the displacement of the point of entry of the loop in the $u$ plane. This gives

$$
\Delta u_{c}=\Delta \rho_{r}\left(\sum_{r} \frac{a_{r}}{i-u_{r}}\right)^{-1}
$$

which changes term $\mathrm{w}$ to read

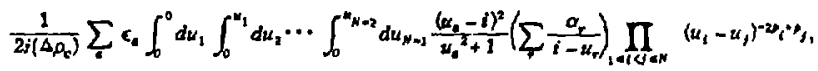

where we have left out the terms $w$ ith $c_{\text {, }}$ in the exponent, since we already have $a$ factor $\epsilon_{x}$.

Unfortunately, this expression is still divergent. This divergence, already seen in the old renormalization calculation, occurs when the loop approaches the boundary of the siring diagram, or in the region of integration where all the $u$ 's are equal. To remedy the situation we introduce another cutofl to climinate this region of integration, and later take the limit as the cut off goes away. We do this in the following way. Since the whole term has a coeffictent linear in.c, the only contributions will come from the region where the integral diverges, 1.e., the region where all but one (at least) of the $u$ 's, say $u_{\text {, }}$, are equal to some value $u_{A}$. Then we have

$$
\sum_{i} \frac{a_{i}}{i-u_{i}}=\frac{a_{k}}{i-u_{k}}+\frac{1}{i-u_{A}} \sum_{n i} a_{r}=\alpha_{\lambda}\left(\frac{1}{i-u_{k}}-\frac{1}{i-u_{A}}\right)
$$

So for the case where $a \neq N$ and $k \neq N$ or $a$, we can write term III in the above region as

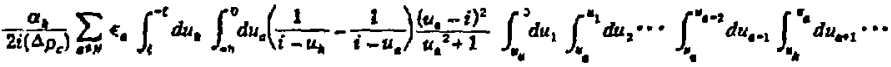

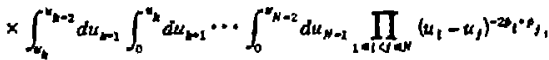

where $\pi \ll<$. The $s_{\text {a }}$ integral has been restricted so that $\left|u_{k}\right|>\xi$. Since $2 l l$ the other $u$ 's are near zero $\left(-u_{x}\right)$, this has the effect of eliminating the region where all the $u^{\prime} \mathrm{s}$ are equal. The $u_{4}$ integral is restricted by $u_{a}>\eta$ (this shosld read $u_{a}<\eta$ if $a<k)$ so that we exclude the region $u_{k}-v_{a}$. The semaining $m$ 's actually have been left unrestricted since there will be no contribution anyway unless they are all near zero. Finally, $y_{A}$ has been set equal to $u_{a}$, which is permissible since all $u_{t}$, $i \neq k$ are equal. It is clear then from (12) that our result is just a constant multiple of $B_{\mu}$. The terms $a=N$ and $k=N$ or $a$, although comewhat different, are similar and give the same result. Also we can easily convince ourselves that changing $u_{c}$ by an infinitesimal amount cannot change our result for term $\mathrm{Il}$ (since it is finite). Thus, we conclude that both terms $\mathrm{I}$ and III simply add to the multiplicative renormalization and do not affect the slope renormalization.

We should point out that we have been using the lact that the $s_{w}$ are all independent. If the number of particles is greater than 26, the number of dimensions, then this is not strictly true. how ever, we note that throughout the derivation of the interacting-string amplitude, no use was made of the number of dimensions. Thus, we would have written down exactly the same expression no matter bow many dimensions we were working in. We, therefore, calculate always in more dimensions than the number of particles we are dealing with, and are confident that the result will be valid in fewer dimensions.

We have now shown tiat the single-loop a mpli. tude for $N$ scalar particles is a slope renormeliza tion. By factorization, we trivially obtain the same result for $N$ excited particles.

Added mote. In a recent paper by Ademollo ef $a l .,{ }^{2}$ the same result has been arrived at. Unlike the above authors, the calculation here is done in the interacting-string picture. The author feels the present work is botli shorter and more 
straightforward, In addition, no explicit use of the appearance of a zero-mass scular particle in the Pomeron sector is made here.

\section{ACKNOWLEDGMENTS}

With creal plessure 1 acknowledge the patience, cosistance and gujdance of Professor S. Mandel- stam throughout the preparation of this work. I would also like to thank him and Hessamaddin Arfaej for carefully reading over the manuscript and suggesting useful additions and corrections. Finally, I would like to thank Warren Siegel for help with a tricky summation.
Work supported by the U. S. Eners Research aud Developmed Agency.

1A. Neveu and J. Scherk, Phys. Rev. D I, 2355 (2970).

For a more complete discustion of this, wee S. Mandel- stum, Plys . Rep. 3C, 259 (1974).

'M. Ademollo, A. D'Add, R, D'Aurle, F. Glioret, E. Nopolituno, S. Scluto, and P. Di Vecehia, Nuel. Phys. B94, 221 (2975). 
III. MONOPOLE PROPAGATOR IN A THEORY WITH CONFINBMENT

\section{A. Theory}

Because quarks have not been seen (or if they have they must still be strongly bound, physicists have been interested in field theories with confined perticles for some time. If particles are permanently confined, then they cannot appear as asymptotlo states. Therefore, no singularities at energles equal to their mass should appear in the S-matrix. One manifestetion of this should be in the behavior of the propagator for the confined particles. All oinBularities at energies equal to their mass should varish. ${ }^{1}$ Thus, for example, the pole thet eppears in the propagetor in the lowest orier of perturbetion theory must somehon be cancelled by higher order corrections. Also because of the confinement, we would expect that for large specelike separations, the propagator should fall off very rapidly.

Here we shall examine the propagetor in a model theory with confinement. We consider the theory with an electromagnetic field $A_{\mu}$ interacting $\mathbf{L}_{\text {th }}$ a scaler Higgs particle $\phi$ and a spin-2 monopole ฟ. The hemiltontan is

$$
\begin{aligned}
\partial \varphi= & \frac{1}{2}\left(\bar{E}^{2}+\bar{H}^{2}\right)+\left|\left(a_{i}-1 e A_{1}\right) \phi\right|^{2}+|\phi|^{2}+e^{2} A_{0}^{2}|\phi|^{2}-\left.u\right|^{2}|\phi|^{2}+ \\
& +\lambda|\phi|^{4}+\bar{\psi}(\bar{\gamma} \cdot(-1 \bar{\gamma}-g \bar{B})+m) \psi,
\end{aligned}
$$


where

$$
\begin{aligned}
& \bar{A}=\bar{A}^{T}+\bar{A}^{B} \\
& \bar{B}=\bar{B}^{T}+\bar{B}^{g} \\
& \bar{H}=\nabla \times \bar{\lambda}^{\mathrm{T}}-\nabla X_{0} \\
& \bar{E}=-\nabla \times \bar{B}^{T}-\nabla A_{0} \\
& A_{0}(x)=i^{\prime} d^{3} x^{\prime} \cdot \frac{e \phi^{*}\left(x^{\prime}\right) \ddot{\partial}_{0} \phi\left(x^{\prime}\right)}{4 \pi|\bar{x}-\bar{x}|} \\
& \chi_{0}(x)=\int a^{3} x^{\prime} \frac{g^{\bar{\psi}}\left(x^{\prime}\right) y_{0} \psi\left(x^{\prime}\right)}{4 \pi\left|\bar{x}-\bar{x}^{\prime}\right|} \\
& \tilde{A}^{g}=g \int d^{3} x^{\prime} \bar{a}\left(\bar{x}-\bar{x}^{\prime}\right) \bar{\psi}\left(x^{\prime}\right) y_{0} \psi\left(x^{\prime}\right) \\
& \bar{g}^{E}=-e \int d^{3} x^{\prime} \bar{a}\left(\bar{x}^{\prime}-\bar{x}\right) \phi^{*}\left(x^{\prime}\right) a_{0} \phi\left(x^{\prime}\right) \\
& \overline{\mathrm{e}}(\overline{\mathrm{x}})=\frac{1}{\overline{8}|2|}\left(\frac{\bar{n} \times \bar{x}}{|\overline{\mathrm{x}}|+\bar{n} \cdot \overline{\mathrm{x}}}-\frac{\overline{\mathrm{n}} \times \overline{\mathrm{x}}}{|\overline{\mathrm{x}}|-\bar{n} \cdot \bar{x}}\right) \text {. }
\end{aligned}
$$

here we have followed the notation of Schwinger. ${ }^{12} A_{\mu}$ and $B_{\mu}$ are the vector potentials for the charge and monopole respectively. $A_{\mu}\left(B_{\mu}\right)$ contains stringlike singularities, Dirac strings, attached to each charge (monopole). We have chosen the monopoles to be rermions because we have querks in the beck of our sind, but the results presented here should be independent of this cholce. Since the $\mu^{2}$ term has the wrong sign for a mass term, the $\phi$ fleld will plck up a vacuum expectation value. This leads to conrinement of the monopoles by the following argument. For finite energy, the term $\left|\left(\partial_{\mu}-1 e A_{\mu}\right)\right|^{2}$ must vanish far from the monopole. Since 
$\phi$ has acquired a vacuum expectation value, we must have

$$
\phi \stackrel{x \rightarrow \infty}{\longrightarrow} k e \int_{x_{0}}^{x} A_{\mu} d x_{\mu}
$$

where $k$ is a constant end $x_{0}$ is some arbitrary point. However, in order for $\phi$ to be rell-defined, we must demand that it be independent of the path of integration. Thus the integral in the exponent, teken around a closed path, must equal $2 \pi \mathrm{m} / \mathrm{e}$. To be specific, let us choose a sirgly charged monopole at the origin with its Dirac string along the z-axis. Then for a path of integration at very large $z$, circling the z-axis and very far from it, we know the integral equals $2 \pi / e$. However, if translate the coutour to large negative $z$, the integral equals $-2 \pi / e$ (for our two-sided string. In any case, the answer is different). Thus there is no way for $\phi$ to be well-defined and continuous in a finite-energy single monopole solution. We conclude that for each monopole there must be an antimonopole at which the Dirac string ends. Thus monopole-antimonopole pairs are confined.

We would like to shift the field $\phi$ by its vacuum expectation value and then examine the higher order corrections to the monopole propegator. However tro technical problems stend in our way. First of all, we have the usual problem that arfaes in all field theories with monopoles. Since the theory only makes sense if eg = 2ma, a perturbation expansion, in both $e$ and $g$ at best carries with it the optimistic hope that after eurmation of 
the series with small e and $g$, a valid analytic continuation can be made to physical values.

However, because we inftially expard in both small $e$ and $g$, to each order in perturbation theory, amplitudes depend on the direction of the Dirac string. We could hope to overcome this difficulty by resuming the series such that, in each step, we add an infinite subset of Feymman diagrams (e.g., Bethe-Selpeter ladders) whose sum is independent of the direction of the Dirac string. To our knowledge, no one (including the present aution) has succeeded in finding even a single aubset of Feynman diagrams independent of the Dirac string. Another possibility is to average over the direction c.. the string (see, for example, Rabl ${ }^{13}$ ), but there is no real reason why this procedure should give correct answers.

The other technical problem is that efter shifting the fleld $\phi$, Fe wind up with a large number of vertices. We might hope that we could generate the properties we expect of the monopole propagator from a few monopole vertices. However, we recall that the proof of confinement depended on the form of the term $\left|\left(\partial_{\mu}-1 e A_{\mu}\right)\right|^{2}$, as well as on $\phi$ having a vacuum expectation value. This would oeem to indicate that most (if not all) of the charged particle vertices need be included too.

Because of all the preceding reesons, the prospects for making progress with this theory are dim. Therefore, we replece the effect of the electromagnetic and charged perticle fields by an effective 
potential for the monopoles. We choose this potential to be lineer by anslogy with the vortex solutions of Nelsen and Olesen ${ }^{14}$ (LandauGinzburg type). They exhibited cylind cally symmetric vortex solutions for the Lagranglan of Eq. I but without the monopole terms. Since the vortices are cylind cally symmetric, their energy is proportional to their length. In the theory with monopoles, the vortices will be finite, since they will end at the monopoles. However, at least for large distances between the monopoles, the energy should still be proportionel to the length of the vortex. Thus we choose an effective potential which is Inear. Our Lagrangian is now

$\widetilde{x}=\bar{\psi}(x)(1 x-m) \psi(x)+\lambda^{2} \int d^{4} y f^{\circ}(x) f^{\circ}(y)|\bar{x}-\bar{y}| \delta\left(t_{x}-t_{y}\right)$

where

$$
j^{\circ}=\bar{\psi}(x) \gamma^{0} \psi(x)
$$

Note that this theory is nonlocal and thus nonrenormalizable. However, this should not matter, since the confinement in our theory is a large distance or infrared effect. Thus a simple cutoff in ail divergent Integrals should sufflce for our purposes.

Ordinarily, we could look at the large $|\bar{x}|$ behevior of the propagator in any spacelike direction. However, since ve now have a nonlocal interaction, we mat confine ourselves to the $t=0$ direction. Thus in the considerations that follow we shall examine the propagator Integrated over $p_{0}$. 
We are now in a position to begin to calculate the monopole propagator. However, It clearly is not feasible to add up all the higher order diagrams. We must devise some approximation scheme. One attractive choice is the set of ladder-like diagrems in Fig. Ia. Throughout this paper we mite the nonlocal four-point interaction as two two-point interactions connected by a dashed line. By crossing symetry they can be rewritten as in Fig. Ib, even though we are no longer in a physical region of the S-matrix. This is a logical choice because we know that, at least in the nonrelativistic case, repeated exchange has been used to create bound states. Thus we could reasonably hope that these ladder-like diagrams would be sufficient to obtain confinement. We shall see later that, at least in the rully relativistic case, this is not the case, and other diagrams need to be considered.

Before beginning the actual calculations, we first exhibit in a concise ray the appraximation we have made. We do this by deriving differential equations for the Green's runctions. This can be done two ways. In the first method, first written dow in a peper by Mandelstam, ${ }^{15}$ we apply the operator $(17$ - If) on

$$
G_{2 n}=\left\langle 0\left|T\left(\bar{\psi}\left(x_{1}\right) \ldots \bar{\psi}\left(x_{n}\right) \psi\left(x_{n+1}\right) \ldots \psi\left(x_{2 n}\right)\right)\right| 0\right\rangle
$$

and use the equations of motion. In the other method, we use the fact that the functional integral of a total functional derivative equals zero, to derive a differential equation that the generating 
functional $Z(J)$ satisfies. 16

$$
0=\int \partial_{\phi} \frac{\delta}{\delta \phi} e^{\left.1\left(S_{i}^{\prime} \phi\right)+J_{\phi}\right)}=\left(\left.\frac{\delta S(\phi)}{\delta \phi}\right|_{\phi=\frac{\delta}{\delta I J}}+J\right) Z(J) .
$$

We next operate -1 th $\left(\frac{\delta}{\delta J}\right)^{n}$ to obtain the same equations as in the rirst method.

The first two equations that we obtain in our present theory are given diagramatically in Fig. 2. Substituting Fig. 2b back into a, we get four different equations depending on how we match up the particles (note that in FiE. $2 \mathrm{~b}$, we can also switch the in particles with the out particles). These are given by Fig. 3. Note that the second term in each of these four equations involves tadpole diagram corrections, which simply lead to a mass renormalization. Thus we can safely ignore them. If we also ignore the last term in Fig. 38 and $d$, we obtain the differentlal equation satiof fed by the sum of the ladder diagrams. This can be seen by repeatedly substituting in. the whole sum where it appears on the right hand side. Therefore, the last term in efther Figure $3 a$ or d (plus its iterations into the third term), which differ only by the inclusion of certain tadpoles, represents all the terms omitted by taing the ladder-like diegrem approximation.

As an aside, neglecting the last term in Fig. 3o or c gives us another series of diagrams that we can sum -- mutiple iterations of single loops. Using the result $A_{1}$ of Eq. A6 in the appendix, 
we have for the sum

$$
\frac{1}{p^{2}-m^{2}+1 \varepsilon} \frac{1}{1-\frac{A_{1}}{p^{2}-m^{2}+1 \varepsilon}}=\frac{1}{p^{2}-m^{2}-A_{1}+1 \varepsilon} \text {. }
$$

This result, not surprisingly, still has olngularities at the ress $m$, and thus this subset of diagrams falls to exhibit confinement.

\section{B. Calculations}

The Bethe-Salfeter equation satisfied by the sum of the ladier diagrams $\psi(p)$ is

$$
\begin{gathered}
\psi(p)=\frac{1}{\phi-m+1 \varepsilon}+\frac{1}{p-m+1 \varepsilon} \int \frac{i d^{4} p^{\prime}}{(2 \pi)^{4}}\left(-8 \pi \lambda^{2} \frac{\left(\bar{p}-\bar{p}^{\prime}\right)^{2}-3 \varepsilon^{2}}{\left(\left(\bar{p}-\bar{p}^{\prime}\right)^{2}+\varepsilon^{2}\right)^{3}}\right) \times \\
\quad \times Y_{0} \psi\left(p^{\prime}\right) \quad \gamma_{0} \frac{1}{\gamma-m+1 \varepsilon}
\end{gathered}
$$

The quantity in the breckets in the above equation is the Fourier transform of our potential $\lambda^{2}|x| \delta(t)$. Letting $G(p)=(p-m+1 \varepsilon) x$ $x \psi(p)(p-m+L E)$, we get

$$
\begin{aligned}
G(p)=p-m & +\int \frac{1 d^{4} p^{\prime}}{(2 \pi)^{4}}\left(-8 \pi \lambda^{2} \frac{\left(\bar{p}-\bar{p}^{\prime}\right)^{2}-3 \varepsilon^{2}}{\left(\left(\bar{p}-\bar{p}^{\prime}\right)^{2}+\varepsilon^{2}\right)^{3}}\right) \times \\
& \times \gamma_{0} \frac{1}{p^{1}-m+1 \varepsilon} G\left(p^{\prime}\right) \frac{1}{p^{\prime}-m+1 f} \gamma_{0} .
\end{aligned}
$$


It is now clear that $G(p)$ must be of the form $G(p)=\gamma_{0} P_{0}+H(\bar{p})$ where $H(\bar{p})$ now depends only on the 3-vector $\bar{p}$. We then can do the $p_{0}^{\prime}$ integral to obtain

$$
\begin{aligned}
& H(\bar{p})=-\bar{y} \cdot \bar{p}-m-\frac{i}{4} \int \frac{i \dot{g}^{3} p^{\prime}}{(2 \pi)^{3}}\left(-8 \pi \lambda^{2} \frac{\left(\bar{p}-\bar{p}^{\prime}\right)^{2}-3 \varepsilon^{2}}{\left(\left(\bar{p}-\bar{p}^{\prime}\right)+\varepsilon^{2}\right)^{3}}\right) \times \\
& \times\left[\frac{2\left(\bar{\gamma} \cdot \bar{p}^{\prime}+m\right)}{\left(\bar{p}^{-2}+m^{2}\right)^{\frac{1}{2}}}+\frac{H\left(\bar{p}^{\prime}\right)}{\left(\bar{p}^{\prime 2}+m^{2}\right)^{2}}-\frac{\left.\left(\bar{y}-\bar{p}^{\prime}+m\right) \gamma^{0} H \bar{p}^{\prime}\right) \gamma^{0}\left(\bar{\gamma} \cdot \bar{p}^{\prime}+m\right.}{\left(\bar{p}^{-2}+m^{2}\right)^{3 / 2}}\right] \text {. }
\end{aligned}
$$

We now write $H(\bar{p})$ in the form

$$
\mathrm{H}(\overline{\mathrm{p}})=\mathrm{J}_{1}(\overline{\mathrm{p}})+\gamma_{0} J_{2}(\overline{\mathrm{p}})+\bar{\gamma} \cdot \overline{\mathrm{p}} \mathrm{J}_{3}(\overline{\mathrm{p}})+\gamma_{0} \bar{\gamma} \cdot \overline{\mathrm{p}} \mathrm{J}_{4}(\overline{\mathrm{p}}) .
$$

Where the $J^{\prime} s$ are numbers, not matrices. We heve not included terms Fith $\gamma_{5}$ beccuse they do not appear in lowest order and they are not generated in higher oriers. After substitution of Eq. 4 into 3, we can separately equate the coefficients of the different $\gamma^{\prime} s$. This leads to

$$
\begin{aligned}
J_{1}(\bar{p}) & =-m+\frac{1}{2} \int \frac{d^{3} p^{\prime}}{(2 \pi)^{3}}-8 \pi \lambda^{2}\left(\frac{\left(\bar{p}-\bar{p}^{\prime}\right)^{2}-3 \varepsilon^{2}}{\left(\left(\bar{p}-\bar{p}^{\prime}\right)^{2}+\varepsilon^{2}\right)^{3}}\right) \times \\
& \times\left[\frac{m}{\left(\bar{p}^{\prime 2}+m^{2}\right)^{\frac{1}{2}}}+\frac{\bar{p}^{\prime 2}\left(J_{1}\left(\bar{p}^{\prime}\right)-m J_{3}\left(\bar{p}^{\prime}\right)\right)}{\left(\bar{p}^{\prime 2}+m^{2}\right)^{3 / 2}}\right] \\
J_{2}(\bar{p}) & =0
\end{aligned}
$$




$$
\begin{aligned}
& \bar{p} J_{3}(\bar{p})=-\bar{y} \cdot \bar{p}+\frac{1}{2} \int \frac{d^{3} p^{\prime}}{(2 \pi)^{3}}\left(-8 \pi \lambda^{2} \frac{\left(\bar{p}-\bar{p}^{\prime}\right)^{2}-3 \varepsilon^{2}}{\left(\left(\bar{p}-\bar{p}^{\prime}\right)^{2}+\varepsilon^{2}\right)^{3}}\right) \times \\
& \times\left[\frac{1}{\left(\bar{p}^{\prime 2}+m^{2}\right)^{\frac{1}{2}}}+\frac{m\left(J_{3}\left(p^{\prime}\right)-m J_{3}\left(\bar{p}^{\prime}\right)\right)}{\left(\bar{p}^{\prime 2}+m^{2}\right)^{3 / 2}}\right] \ddot{p}^{\prime} \\
& \bar{p} J_{4}(\bar{p})=\frac{1}{2} \int \frac{d^{3} \bar{p}^{\prime}}{(2 \pi)^{3}}\left(-8 \pi \lambda^{2} \frac{\left(\bar{p}-\bar{p}^{\prime}\right)^{2}-3 \varepsilon^{2}}{\left((\bar{p}-\bar{p})^{2}+\varepsilon^{2} \bar{j}^{3}\right.}\right) \times \\
& \times \frac{J_{4}\left(\bar{p}^{\prime}\right)}{\left(\bar{p}^{\prime 2}+m^{2}\right)^{\frac{3}{2}}} \bar{p}^{\prime}
\end{aligned}
$$

We can at this point set $J_{4}(\bar{p})=0$, since 1t does not appear fn lowest order, and From Eq. 5d eech succeeding term is zero. Equation 5a and c remain. We do not know how to solve them, but in the Appendix we place an upper limit on the large $r$ behevior of the solution. However, we can show that no solution exists such that the singularity of the propagator at the mass of the monopale has disappeared. Since we are interested in large space like separations at $t=0$, we examine

$$
\begin{aligned}
& \left.\int d p_{0} \psi(p)=\int d p_{0} \frac{1}{p-m+1 \varepsilon} i \gamma_{0} p_{0}+H(\bar{p})\right) \frac{1}{p-m+1 \varepsilon}= \\
& =\int \frac{d p_{0}}{\left(p^{2}-m^{2}+1 \varepsilon\right)^{2}}(p+m)\left(Y_{0} p_{0}+J_{1}(\bar{p})+\bar{\gamma} \cdot \bar{p} J_{3}(\bar{p})\right)(p+m)= \\
& =-\frac{\pi}{\left(\bar{p}^{2}+m^{2}\right)^{3 / 2}}\left\{\left[J_{1}(\bar{p})-m J_{3}(\bar{p})+\frac{m}{\bar{p}^{2}}\left(\bar{p}^{2}+m^{2}\right)\right]+\right. \\
& \text { (Equation } 6 \text { continued on next page) }
\end{aligned}
$$




$$
\left.+m \bar{\gamma} \cdot \bar{p} J_{1}(\bar{p})-m J_{3}(\bar{p})-\frac{1}{m}\left(\bar{p}^{2}+m^{2}\right)\right\} \text {. }
$$

In doing the $p_{0}$ integral, we have ignored a term, formelly infinite, but odd in $p_{0}$. In order for the result in Eq. 6 to be regular at $\bar{p}^{2}=-m^{2}$, we must have both

$$
\frac{I}{\left(\bar{p}^{2}+m^{2}\right)^{3 / 2}}\left[J_{2}(\bar{p})-m J_{3}(\bar{p})+\frac{m}{\bar{p}^{2}}\left(\bar{p}^{2}+m^{2}\right)\right]
$$

and

$$
\frac{1}{\left(p^{2}+m^{2}\right)^{3 / 2}}\left[J_{1}(\bar{p})-m J_{3}(\bar{p})-\frac{1}{m}\left(\bar{p}^{2}+m^{2}\right)\right]
$$

regular. However, this is impossible because their difference is singular. Therefore, if a solution exists at all, it contains a singularity at the mass of the monopole. Thus we have failed to exhibit confinement. We believe this is due to our inclusion or ladter diagrans with pair production (Fig. 4b), but not the corresponding crossed diagram (Fig. 4c). These diagrams also contribute to the binding "forces" on the monopoles and should be important. Unfortunately, we know of no wey to correctly take them into account. However, in the nonrelativistic approximation, there is no pair creation, and the types of diagrams represented by both Fige. $4 b$ and c are absent (remember we have an instanteneous interaction) and only those of Fig. 4a remain. Thus we might hope to obtain conflement in this approximation, and we examine this possibility next. 


\section{Non-relativistic approximation}

Starting with our Bethe-Salpeter Eq. 2, we can do the $p^{0^{\prime}}$ integration on the right hand side. Defining

$$
\begin{aligned}
& \phi(\bar{p})=\int_{-\infty}^{\infty} d p^{\circ} \psi(p) \\
& H_{a}=\gamma_{0}(\bar{\gamma} \cdot \bar{p}+m j \\
& H_{b}=\gamma_{0}(-\bar{Y} \cdot \bar{p}+m)
\end{aligned}
$$

7e have

$$
\begin{aligned}
\left(p^{0}-a_{a}(\bar{p})\right) \psi(p)\left(p^{0}-H_{b}(\bar{p})\right)= & \gamma^{0} p^{0}+\bar{Y} \cdot \bar{p}+ \\
& +\int \frac{1 d^{3} p^{\prime}}{(2 \pi)^{4}}\left(-8 \pi \lambda^{2} \frac{\left(\bar{p}-p^{\prime}\right)^{2}-3 \varepsilon^{2}}{\left(\left(\bar{p}-\bar{p}^{\prime}\right)^{2}+\varepsilon^{2}\right)^{3}}\right)
\end{aligned}
$$

We can now treat the $4 \times 4$ matrix $\psi(p)$ as a wave function in the product space of two spinor particles. We proceed according to the rethod of Salpeter 27 for treating instentaneous interactions and make the following definitions

$$
\Lambda_{ \pm}^{a}=\frac{E_{B}(\bar{p})+H_{B}(\bar{p})}{2 E_{a}(\bar{p})}
$$

where $E_{a}(\bar{p})=\left(\bar{p}^{2}+m^{2}\right)^{2}$ 
end similarly for particle b. In addition, we define

$$
\begin{aligned}
& \psi_{++}(p)=\Lambda_{+}^{a}(\bar{p}) \psi(p) \Lambda_{+}^{b}(\bar{p}) \\
& \psi_{+-}(p)=\Lambda_{+}^{a}(\bar{p}) \psi(p) \Lambda_{-}^{b}(\bar{p}), \text { etc. }
\end{aligned}
$$

Then we arrive at

$$
\begin{aligned}
& F_{++}(p) \psi_{++}(p)=\Lambda_{+}^{\theta}(\bar{p}) \Gamma(p) \Lambda_{+}^{b}(\bar{p}) \\
& F_{+-}(p) \psi_{+-}(p)=\Lambda_{+}^{a}(\bar{p}) \Gamma(p) \Lambda_{-}^{b}(\bar{p}), \text { etc. }
\end{aligned}
$$

where $F_{++}(p)=\left(p^{0}-E_{a}(\bar{p})+i \varepsilon\right)\left(p^{0}-E_{b}(\bar{p})+i \varepsilon\right)$

$$
F_{+-}(p)=\left(p^{0}-E_{a}(\bar{p})+i \varepsilon\right)\left(p^{0}+E_{b}(\bar{p})-i \varepsilon\right) \text {, etc. }
$$

and $\Gamma(p)$ is the right hand side of Eq. 7. He now divide each of Eq. 8 by the appropriate $F(p)$ and integrate over $p^{0}$ using

$$
\begin{aligned}
& \int_{-\infty}^{\infty} d p^{0}\left(p^{0}+a \mp i \varepsilon\right)^{-1}\left(p^{0}+b \pm i \varepsilon\right)^{-1}= \pm 2 \pi i(b-a)^{-1} \\
& \int_{-\infty}^{\infty} d p^{0}\left(p^{0}+a \pm i \varepsilon\right)^{-1}\left(p^{0}+b \pm i \varepsilon\right)^{-1}=0 \\
& \int_{-\infty}^{\infty} d p^{0} p^{0}\left(p^{0}-a+i \varepsilon\right)^{-1}\left(p^{0}-b+i \varepsilon\right)^{-1}=-\pi I \\
& \int_{-\infty}^{\infty} d p^{0} p^{0}\left(p^{0}+a-i \varepsilon\right)^{-1}\left(p^{0}-b+i \varepsilon\right)^{-1}=\pi \frac{a-b}{a+b} .
\end{aligned}
$$


In the last two equations, we have thrown away a term, formally infinite, but odd in $p^{0}$. This leads to

$$
\begin{aligned}
& -\left(E_{a}(p)+E_{b}(\bar{p})\right) \phi_{-+}(\bar{p})=A_{-}^{a}(\bar{p})\left[\pi l\left(E_{b}(\bar{p})-E_{a}(\bar{p})\right) y_{0}+2 \pi i(\bar{\gamma} \cdot \bar{p}-m)-\right. \\
& \left.-\int \frac{i d^{3} p^{\prime}}{(2 \pi)^{4}}\left(-8 \pi \lambda^{2} \frac{\left(\bar{p}-\bar{p}^{\prime}\right)^{2}-z^{2}}{\left((\bar{p}-\bar{p})^{2}+\varepsilon^{2}\right)^{3}}\right) \phi\left(\bar{p}^{\prime}\right)\right] \times \\
& \times \Lambda_{+}^{b}(\bar{p}) \\
& -\left(E_{a}(\bar{p})+E_{b}(\bar{p}) h_{+-}(\bar{p})=\Lambda_{+}^{a}(\bar{p})\left\{\pi i \left(E_{a}(\bar{p})-E_{b}(\bar{p}) h_{\gamma_{0}}+2 \pi i(\bar{\gamma} \cdot \bar{p}-m)-\right.\right.\right. \\
& \left.-\int \frac{i d^{4} p^{\prime}}{(2 \pi)^{3}}\left(-8 \pi \lambda^{2} \frac{\left(\bar{p}-\bar{p}^{\prime}\right)^{2}-3 \varepsilon^{2}}{\left(\left(\bar{p}-\bar{p}^{\prime}\right)^{2}+\varepsilon^{2}\right)^{3}}\right) \phi(\bar{p})\right] \Lambda_{-}^{b}(\bar{p}) \text {, } \\
& -\phi_{++}(\bar{p})=\pi \dot{L} \Lambda_{+}^{a}\left(\bar{p} h_{0} \Lambda_{+}^{b}(\bar{p}),\right. \\
& \phi_{--}(\bar{p})=\pi \Lambda_{-}^{a}\left(\bar{p} \gamma_{0} \Lambda_{-}^{b}(\bar{p}) .\right.
\end{aligned}
$$

Since in our case, $E_{a}-E_{b}=0$, ve have

$$
\begin{aligned}
& \left(H_{Q}(\bar{p})-H_{b}(\bar{p})\right) \phi(\bar{p})=\left(\Lambda_{-}^{z}(\bar{p}) \Lambda_{+}^{b}(\bar{p})-\Lambda_{+}^{a}(\bar{p}) \Lambda_{-}^{b}(\bar{p})\right)[2 \pi(\bar{\gamma} \cdot \bar{p}-m)- \\
& \left.-\int \frac{i d^{3} p^{\prime}}{(2 \pi)^{3}}\left(-8 \pi \lambda^{2} \frac{\left(p-p^{\prime}\right)^{2}-3 \varepsilon^{2}}{\left(\left(p-p^{\prime}\right)^{2}+\varepsilon^{2}\right)^{3}}\right) \phi\left(p^{\prime}\right)\right] \text {, }
\end{aligned}
$$


where for convenience we have written the $b$ operators on the left, even though they really should appear on the right. Eq. 9 looks different than the corresponding equation in Salpeter's ${ }^{17}$ article. This Is due to the fact that our $\phi(\bar{p})$ is a wave function for a particle and entiparticle, whereas his is for two particles. To remedy this we multiply by the charge conjugation operator $C$ on the right to obtain

$$
\begin{aligned}
& -\left(\mu_{a}(\bar{p})+H_{b}^{T}(\bar{p})\right) \phi^{c}(\bar{p})=\left(\Lambda_{+}^{a}(\bar{p}) \Lambda_{+}^{b} T(\bar{p})-\Lambda_{-}^{a}(\bar{p}) \Lambda_{-}^{b}(\bar{p})\right) \times \\
& \times\left[2 \pi i(\bar{\gamma} \cdot \bar{p}-m) c-\int \frac{d^{3} k}{(2 \pi)^{3}} \times\right. \\
& \left.\times\left(-8 \pi \lambda^{2} \frac{\left(\bar{p}-\bar{p}^{\prime}\right)^{2}-3 \varepsilon^{2}}{\left((\bar{p}-\bar{p})^{2}+\varepsilon^{2}\right)^{3}}\right) \phi^{c}\left(\bar{p}^{\prime}\right)\right)
\end{aligned}
$$

where $\phi^{c}(\bar{p})=\phi(\bar{p}) C$. In the nonrelativistic Iimit the factor involving the $\Lambda^{\prime} \mathrm{s}$ equals one, and all the homogeneous terms in $\phi$ reduce to the Schroedinger Hamiltonian operator acting on the "large" part of the wave function $\phi_{*+}(\bar{p})$. In coordinate space we then have

$$
\mathrm{H}_{s}(\bar{x}) \phi^{c}(\bar{x})=2 \pi i(-1 \bar{\gamma} \cdot \nabla-m) 6^{3}(\bar{x}) c
$$

where $H_{\varepsilon}(\bar{x})=-\frac{1}{2 m} \nabla^{2}+\lambda^{2}|\bar{x}|$.

For $\bar{x} \neq 0$, the right hand side in $\mathrm{Eg}$. 11 equals zero. The problem becomes simply thet of finding the large $\bar{x}$ behavior of the 
Schrödinger wave function with energy $E=0$. (Boundary conditions at the origin, which quantize the allowed energies, do not apply here). The angular part of the equation can be separated out in the standard way for a central potential. We are then left with the radial equation

$$
\left(\frac{d^{2}}{d r^{2}}-2 m \lambda^{2} r-\frac{\ell(\ell+1)}{r^{2}}\right)\left(r R_{\ell}(r)\right)=0 .
$$

For large $I$, we can ignore the angular momentum term compared to the potential. Resceling $r$, we arrive at the Airy differentlal equation. The solution has the asyunptotic form

$$
R_{\ell}(r)=\frac{1}{r} \operatorname{Ai}\left(\left(2 m \lambda^{2}\right)^{1 / 3} r\right) \stackrel{r \rightarrow \infty}{\longrightarrow} \frac{\sqrt{\pi}}{2\left(2 m \lambda^{2}\right)^{1 / 12}} \frac{\exp \left(-\frac{2}{3} \sqrt{2 m \lambda^{2}} r^{3 / 2}\right)}{r^{3 / 2}}
$$

where, as usual, we have discaried the exponentlally increasing solution. This shows thet our wave function, and consequently the propagator, falls off much faster than the free propagator. In fact, since it falls off faster than $e^{-m}$ for ans $m$, ail singulerities in momentum spece must be absent ${ }^{19}$ and we have finally exhibited confinement. 


\section{ACKNOWLEDGEMENTS}

I would like to thank Professor Stanley Mandelstam for his continual assistance and guidance throughout the period of my graduete research.

Thanks are also due to my parents, without whom I would never achieved what I have. 


\section{APPENDIX}

As we mentioned earlier, we have not been able to find the large $\bar{x}$ behavior of the solution to the Bethe-Salpeter equation for the propagator. However, at least in the case of bosonic monopoles, and in the context of the approxination scheme beion, we can show that any solution must fall of faster then the free propagator.

The idea is to directiy calculate the ladder diagrams by iteration in the leading term approximation. Given the amplitude for the $n$ rung diagram, it is easy to write down a single integrel expression for the $n+1$ rung diagram, using the fact that 1 t has an $n$ rung subdiagram. We start at the single loop level and at each level keep only the most singular term at $\vec{p}^{2}=-m^{2}$. We can hope to derive a simple expression for the amplitude as a function of $\mathrm{n}$. The problem with this procedure is that more of ten than not, the integrals simp, become more and more complicated at higher orders and no pettern emerges. We shell see that for bosonic monopoles we are lucky and the procedure works.

The single loop diagram for a bosonic monopole is given by

$$
\begin{gathered}
A_{1}=\frac{1}{\left(p^{2}-\bar{m}^{2}+1 \varepsilon\right)^{2}} \int \frac{1 d^{4} p^{1}}{(2 \pi)^{4}} \frac{\left[-\left(2 p_{0}+p_{0}^{\prime}\right)^{2}\right]}{(p+p)^{2}-m^{2}+i \varepsilon} \times \\
\left(-8 \pi \lambda^{2} \frac{\bar{p}^{-12}-3 \varepsilon^{2}}{\left(\bar{p}+\varepsilon^{2}\right)^{3}}\right) .
\end{gathered}
$$


The quadratic term in $P_{0}$ is due to the derivative coupling. Introducing Feynman parameters, we have

$$
\begin{aligned}
& A_{1}=-\frac{31 \lambda^{2}}{\pi^{3}} \frac{1}{\left(p^{2}-m^{2}+1 \varepsilon\right)^{2}} \int d p_{0}^{\prime}\left(p_{0}^{2}+p_{0}^{\prime 2}\right) \int d x_{1} \ldots d x_{4} \delta\left(1-\sum_{n=1}^{4} x_{n}\right) x \\
& \times \int d^{3} p^{\prime} \frac{\bar{p}^{\prime 2}-3 \varepsilon^{2}}{\left[\bar{p}^{\prime 2}+2 x_{1} \bar{p} \cdot \bar{p}-x_{1}\left(p_{0}^{2}-m^{2}-\bar{p}^{2}+i \varepsilon+\varepsilon^{2}\right)+\varepsilon^{2}\right]^{4}} \\
& =-\frac{3 \lambda^{2}}{16 \pi} \cdot \frac{1}{\left(p^{2} \cdot-m^{2}+1 \varepsilon\right)^{2}} \int d p_{0}^{\prime}\left(p_{0}^{2}+p_{0}^{2}\right)\left(-\frac{2}{3} \frac{\partial}{\partial a}\right) \\
& \times\left.\int_{0}^{1} d x \frac{\left(1-x_{1}\right)^{2}}{\sqrt{\left.x_{1}^{2} p^{2}+\left(p_{0}^{2}-m^{2}-p^{-2}+1 \varepsilon+\varepsilon^{2}\right)+\varepsilon^{2}(2 \alpha-3)\right]^{3 / 1}}}\right|_{\alpha=1} ^{\infty} \\
& =\frac{\lambda^{2}}{\pi} \frac{1}{\left(p^{2}-m^{2}+1 \varepsilon\right)^{2}} \int_{-\infty}^{\infty} d p_{0}^{\prime}\left(p_{0}^{2}+p_{0}^{2}\right) \frac{\left(p_{0}^{\prime 2}-m^{2}+1 F\right)^{\frac{1}{2}}}{\left(p_{0}^{2}-p^{2}-m^{2}+1 \varepsilon\right)^{2}} \\
& =-\left.\frac{2 \lambda^{2}}{\pi} \frac{1}{\left(p^{2}-m^{2}+1 \varepsilon\right)^{2}} \frac{\partial}{\partial \alpha} \int_{0}^{\Lambda / m} d p_{0}^{1} \frac{\left(p_{0}^{2}-1+i \varepsilon\right)^{\frac{1}{2}}}{\alpha p_{0}^{2}+(\alpha-1)\left(\frac{p o}{m}\right)^{2}-\left(\frac{p}{m}\right)^{2}-1+i \varepsilon}\right|_{a=1}
\end{aligned}
$$

To obtain Eq. A2, we also shifted the $P_{0}^{\prime}$ Integral. In arriving at Eq. (A3), we did the $\overline{\mathbf{p}}^{\prime}$ Integral using

$$
\int \frac{a^{3} x\left(\bar{x}^{2}+a\right)}{\left[\bar{x}^{2}+2 \bar{b} \cdot \bar{x}+c-1 \varepsilon\right]^{4}}=\frac{\pi I}{8} \frac{a+c}{\left(b^{2}-c+1 \varepsilon\right)^{5 / 2}}
$$

in addition to the $x_{2}, x_{3}, x_{4}$ integrals. To get Eq. (AK), we used 


$$
\begin{aligned}
& \int \frac{d x}{\pi^{3}}=\frac{2(2 c x+b)}{\Delta \sqrt{R}} \\
& \int \frac{x d x}{\pi^{3}}=-\frac{2(2 a+b x)}{\Delta \sqrt{R}} \\
& \int \frac{x^{2} d x}{\sqrt{R^{3}}}=-\frac{\left(\Delta-b^{2}\right) x-2 a b}{\Delta \sqrt{R}}
\end{aligned}
$$

where $R=a+b x+c x^{2}$ and $\nabla=4 e c-b^{2}$, set $a=1$, and then took the limit $\varepsilon=0$. Surprisingly, all divergent terins cancel. In Eq. As, we have introduced a cutoff in the divergent $p_{0}^{\prime}$ integral. The integral appearing in Eq. A5 is a mess to do. We must integrate separately the regions above and below one. Each of these can be done by a messy substitution $\left(p_{0}^{\prime}=\frac{1 \pm x^{2}}{1 \mp x^{2}}\right)$ and a lot of algebra. We thus arrive at the result

$$
\begin{aligned}
A_{1}= & \frac{2 \lambda^{2}}{\pi} \frac{1}{\left(p^{2}-m^{2}+1 \varepsilon\right)^{2}}\left[\ln \frac{2 \Lambda}{m}-\frac{\pi l}{2}+\left(\frac{|\bar{p}|}{\left(\bar{p}^{2}+m^{2}\right)^{\frac{1}{2}}}+\frac{m^{2}\left(p_{0}^{2}+\bar{p}^{2}+m^{2}\right)}{2|\bar{p}|\left(\bar{p}^{2}+m^{2}\right)^{3 / 2}}\right) \times\right. \\
& \left.\times \ln \left(\frac{\left(\bar{p}^{2}+m^{2}\right)^{\frac{1}{2}}-|\bar{p}|}{m}\right)-\frac{p_{0}^{2}+\bar{p}^{2}+m^{2}}{2\left(\bar{p}^{2}+m^{2}\right)}\right] .
\end{aligned}
$$

Since we are interested only in the large $\bar{z}$ behevior, we need only keep the most singular term at the closest singularity to the real axis, which turns out to be at $|\bar{p}|= \pm 1 m$. This leads to 
$A_{1} \longrightarrow \frac{\lambda^{2}}{\pi} \frac{1}{\left(p^{2}-m^{2}+1 \varepsilon\right)^{2}} \frac{m^{2} p_{0}^{2}}{\left(\bar{p}^{2}+m^{2}\right)^{3 / 2}} \quad\left[\frac{\ln \left(\frac{\left(\bar{p}^{2}+m^{2}\right)^{\frac{1}{2}}-|\bar{p}|}{m}\right)}{|\bar{p}|}\right]$

The singularity at $|\bar{p}|= \pm$ im inside the logaritim is quite reak compared to that of the $\left(\bar{p}^{-2}+m^{2}\right)^{-3 / 2}$ factor, and ignoring it ahould not affect the answer much. We do this for simplicity. Thus we evaluate the term in the square brackets at $|\bar{p}|=$ in. In either case we get $-\frac{\pi}{2 m}$. Our result is now

$A_{1} \longrightarrow-\frac{\lambda^{2} m p_{0}^{2}}{2\left(\bar{p}^{2}+m^{2}\right)^{3 / 2}} \frac{1}{\left(p^{2}-m^{2}+1 \varepsilon\right)^{2}}$

Once we have $A_{n}$, we get $A_{n+1}$ as follows:

$A_{n+I}=-\int \frac{i d^{4} p^{\prime}}{(2 \pi)^{4}}\left(p_{0}+p_{0}^{\prime}\right)^{2}\left(-8 \pi \lambda^{2} \frac{\left(\bar{p}-\bar{p}^{\prime}\right)^{2}-3 \varepsilon^{2}}{\left(\left(\bar{p}-p^{\prime}\right)^{2}+\varepsilon^{2}\right)^{3}}\right) A_{n}$

Now suppose $A_{n}$ is of the form

$$
A_{n}=\frac{k p_{0}^{2}}{\left(p^{2}+m^{2} r\right.} \frac{1}{\left(p^{2}-m^{2}+1 \varepsilon\right)^{2}}
$$

where $z$ is a constant.

Then re can to the $p_{0}^{\prime}$ integral

$\int_{-\Lambda}^{h} \frac{d p_{0}^{\prime}\left(p_{0}+p_{0}^{\prime}\right)^{2} p_{0}^{\prime 2}}{\left(p_{0}^{\prime 2}-\bar{p}^{2}-m^{2}+1 \varepsilon\right)^{2}}=\lambda-\frac{\pi i}{2} \frac{3\left(\bar{p}^{2}+m^{2}\right)+p_{0}^{2}}{\left(\bar{p}^{2}+m^{2}\right)^{\frac{1}{2}}} \rightarrow-\frac{\pi 1}{2} \frac{p_{0}^{2}}{\left(\bar{p}^{2}+m^{2}\right)^{\frac{1}{2}}}$

where in the last step we have kept only the most singular term 
at $|\vec{D}|= \pm i m$. Therefore

$$
\begin{gathered}
A_{n+1}=\frac{\lambda^{2} z p_{0}^{2}}{4 \pi^{2}} \frac{1}{\left(p^{2}-m^{2}+1 \varepsilon\right)^{2}} \int \frac{d^{3} p^{1}}{\left(\bar{p}^{-12}+m^{2}\right)^{5}+\frac{1}{2}}\left(\frac{\left(\bar{p}-\bar{p}^{1}\right)^{2}-3 \varepsilon^{2}}{\left((\bar{p}-\bar{p})^{2}+\varepsilon^{2}\right)^{3}}\right) \\
\longrightarrow-\frac{\lambda^{2} k\left(r+\frac{1}{2}\right) m p_{0}^{2}}{2\left(\bar{p}^{2}+m^{2}\right)^{3+3 / 2}} \frac{1}{\left(p^{2}-m^{2}+1 \varepsilon\right)^{2}}
\end{gathered}
$$

where we again have kept only the most singular term, this time by Fourler transforming to position space, taking: the limft $|\bar{x}|+\infty$, and then Fourier transforming back. We have been fortunate. The result of Eq. (ALO) is again of the form of (A9). Since (AI) is also of this form (Eq. A7), all $A_{n}$ are of this form. In fact it is not hard to see that

$$
\begin{aligned}
\mathbf{r} & =\frac{3}{2} n \\
A_{n} & =-\frac{3 \lambda^{2}\left(n-\frac{2}{3}\right) m}{4\left(\bar{p}^{2}+m^{2}\right)^{3 / 2}} A_{n-1} \\
& =\left(-\frac{3 \lambda^{2} m}{4\left(\bar{p}^{2}+m^{2}\right)^{3 / 2}}\right)^{n-1} \frac{\Gamma\left(n+\frac{1}{3}\right)}{\Gamma\left(\frac{4}{3}\right)} A_{n-1} \\
& =\frac{2}{3} \frac{p_{0}^{2}}{\left(p^{2}-m^{2}+1 \varepsilon\right)^{2}}\left(-\frac{3 \lambda^{2} m}{4\left(\bar{p}^{2}+m^{2}\right)^{372}}\right)^{n} \frac{\Gamma\left(n+\frac{1}{3}\right)}{\Gamma\left(\frac{4}{3}\right)} .
\end{aligned}
$$

If we try to sum up the $A_{n}$ we have obtained, the series diverges. Instead we Fourier transform to position space, keeping only the leading term for large distance (and time $t=0$, for simplicity). 
This leads to

$$
\begin{gathered}
\sum_{n=1}^{\infty} A_{n} \stackrel{r+\infty}{\longrightarrow} \sum_{n=1}^{\infty} \frac{2}{3}\left(-\frac{1}{4}\right)\left(-\frac{3 \lambda^{2} m}{4}\right)^{n} \frac{e^{-m r}}{4 \pi \Gamma\left(\frac{3}{2} n+\frac{1}{2}\right)}\left(\frac{\left.r^{\frac{3}{2}}\right)^{\frac{1}{2}}}{2 \pi}\right)^{\frac{\Gamma\left(n+\frac{1}{3}\right)}{\Gamma\left(\frac{4}{3}\right)}} \\
=\frac{1 \sqrt{2 \pi} e^{-m r}}{12 r^{3 / 2} \Gamma\left(\frac{4}{3}\right)}\left(\frac{3 \lambda^{2} m}{4}\left(\frac{r}{2 m}\right)^{3 / 2}\right) \sum_{n=0}^{\infty} \frac{\Gamma\left(n+\frac{4}{3}\right)}{\Gamma\left(\frac{3}{2} n+2\right)} \times\left(-\frac{3 \lambda^{2} m}{4}\left(\frac{r}{2 m}\right)^{3 / 2 i n}\right. \\
\stackrel{r+\infty}{\longrightarrow} \frac{i}{8 \pi} \sqrt{\frac{2 m}{\pi}} \frac{e^{-m r}}{r^{3 / 2}},
\end{gathered}
$$

where, in the last steps, we have used a theorem of Wright. ${ }^{28}$ To the result of Eq. (All) we must add the Fourier transform of the free propagator (at $t=0$ )

$$
\int \frac{d^{4} p}{(2 \pi)^{4}} \frac{e^{i \bar{p} \cdot \bar{x}}}{p^{2}-m^{2}+1 \varepsilon} \stackrel{r \rightarrow \infty}{\longrightarrow}-\frac{1}{8 \pi} \sqrt{\frac{2 m}{\pi}} \frac{e^{-m r}}{r^{3 / 2}} .
$$

As we can see, the sum equals zero, and the leading terms cancel. This would suggest that we should look at nonleading terms. This cannot be done in the context of this calculation without the algebrc becoming unmanageable. Therefore all we can say is that our propagator should fall off faster than the free propagator. 


\section{FOOTNOTES AND REFERENCES}

1. G. Veneziano, Nuovo Cimento 57A, 190 (1968)

2. G. Venezlano, Phys. Reports 9, 4 (1974); C. Reblif, Phys. Reports 12, 1 (1974); S. Mandelstam, Phys. Reports 13C, 259 (1974); J. Scherk, Rev. Mod. Phys. 47, I (1975); P. Frempton, "Dusl Resonence Models," W. A. Benjamin, 1974.

3. K. Bardakct and H. Ruegg, Phys. Rev. 181, 1884 (1969);

H. M. Chan and S. T. Asou, Phys. Letters 28B, 485 (1969); C. G. Goebel and B. Sakita, Phys. Rev. Letters 22, 257 (1969); 2. Koba and H. B. Nielsen, Nuclear Physies B1O, 633 (1969).

4. S. Fubini, D. Gordon, and G. Venezieno, Pryss. Letters $29 B$ 679 (1969); Y. Nambu, Proceedings of the International Conference on Symmetries end Quark Models, Hayne State University (1969); L. Sussikind, Phys. Rev. DI, 1182 (1970).

5. R. C. Brower, Phys. Rev. D6, 1655 (1972).

6. H. B. Nielsen, Proceedings of the XVth International Conference on High-Energy Physics, Kiev (1970); L. Susskind, Nuovo Cimento $69 A, 457(1970)$.

7. C. S. Hsue, B. Sekita, and M. A. Virasoro, Phys. Rev. D2, 2857 (197C); J-L. Gervais and B. Sakita, Fays. Rev. Letters 30, 716 (1973); S. Mandelstam, Nuclear Physics B64, 205 (1973).

B. D. J. Gross, A. Neveu, J. Scherk, and J. H. Schwarz, Phys. Rev. D2 697 (1970); C. Iovelace, Phys. Ietters 34B, 500 (1970). 
9. A. Neveu and J. H. Schwarz, Nuclear Physics B31, 86 (1971).

10. A. Neveu and J. Scherk, Phys. Rev. DI, 2355 (1970).

11. One might object that in two dimensional QCD this does not happen. Rather the quarks decouple when put on mass shell. However, if this were the case here, we would expect the propagator to have a pole, not a cut at the mass of the monopole. He find no such behevior.

12. J. Schwinger, Phys. Rev. 144, 1087 (1966).

13. A. Rabl, Phys. Rev. I79, 1363 (1969).

14. H. B. Nielsen and P. Olesen, Nuclear Physics B61, 45 (1973).

15. S. Mandelstam, Phys. Rev. 175, 1580 (1968).

16. I am indebted to Werren Siegel for pointing this out to me.

17. E. E. Salpeter, Phys. Rev. 87, 328 (1952).

18. E. M. Wright, J. London Math. Soc. 10, 287 (1935);

Proc. Iondon Math. Soc. 46, 389 (1940).

19. This follows from Theorem IX.13, p. 18 in M. Reed and B. Simon, "Methods of Modern Mathematics, II: Fourier Analysis, SelfAdjointness," Acadenic Press (1975). 


\section{FIGURE CAPTIONS}

Fig. 1: Ladder diagrams.

Fig. 2: Differential equations satiafled by the Green's functions.

Fig. 3: $\quad$ Substitutions of Figure 20 into 28

Fig. 4: Diagrams neglected in verious apreoxinations. 
$-37-$
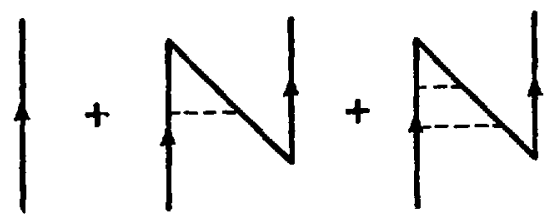

(a)
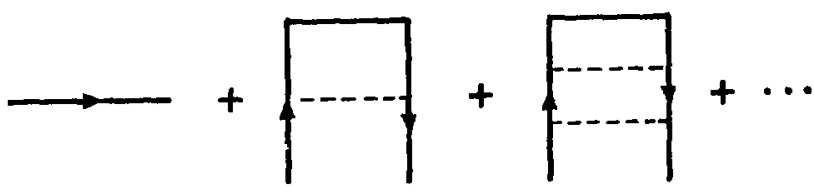

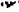

(b)

XBL 776-1187

Fig. 1

ronom 
$-38-$

อ
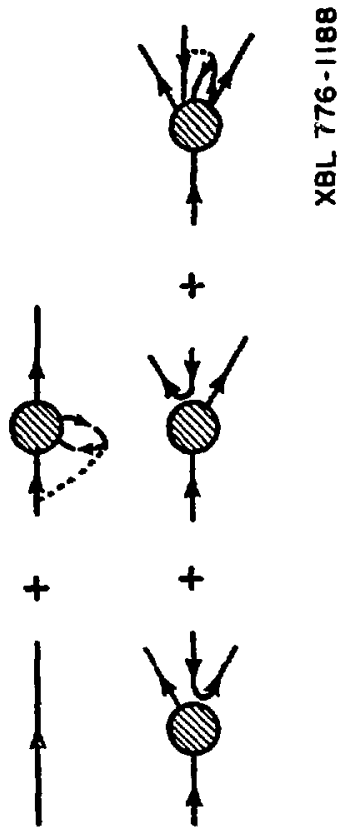

Nis

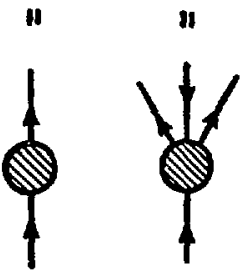




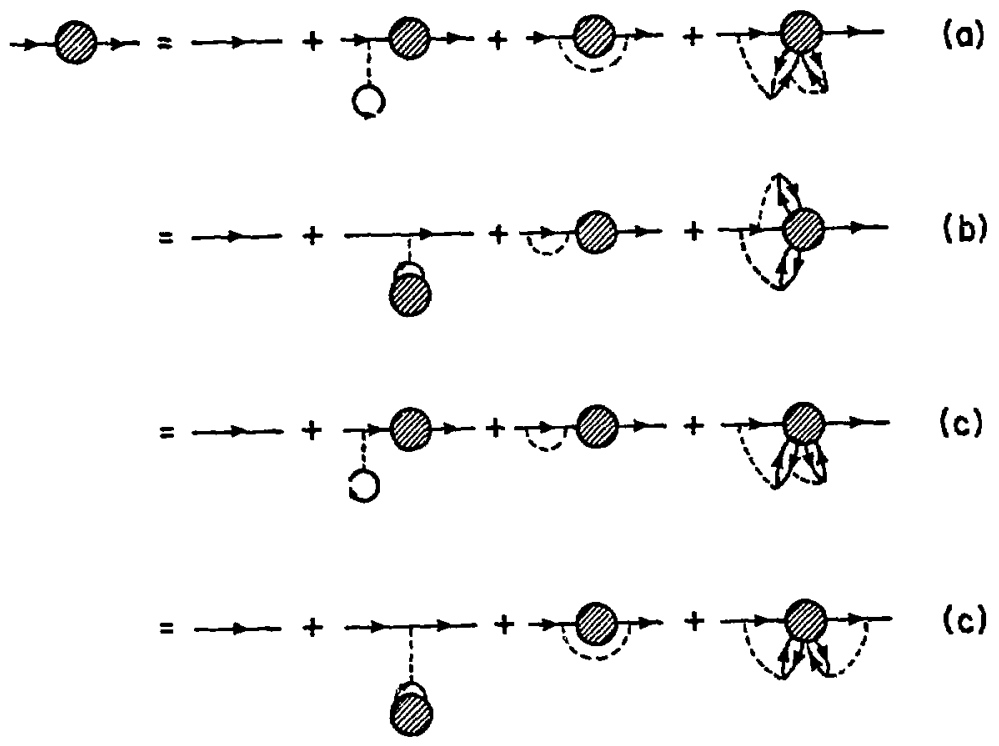

XBL $776-1190$

Fig. 3 
$-40$

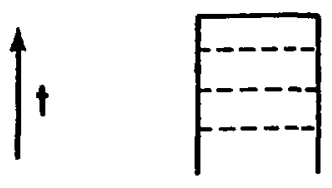

(a)

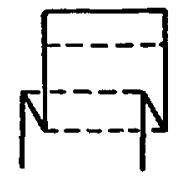

(b)

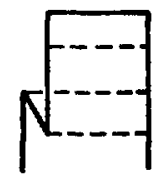

(c)

XBL 776-1189

Fig. 4 